\title{
Association of the miR-149 Rs2292832 Polymorphism with Papillary Thyroid Cancer Risk and Clinicopathologic Characteristics in a Chinese Population
}

\author{
Wen-Jun Wei ${ }^{1,2, \dagger}$, Zhong-Wu Lu ${ }^{1,2, \dagger}$, Duan-Shu Li ${ }^{1,2}$, Yu Wang ${ }^{1,2}$, Yong-Xue Zhu ${ }^{1,2}$, \\ Zhuo-Ying Wang ${ }^{1,2}$, Yi Wu ${ }^{1,2}$, Yu-Long Wang ${ }^{1,2, *}$ and Qing-Hai Ji ${ }^{1,2, *}$
}

1 Department of Head \& Neck Surgery, Cancer Hospital, Fudan University, Shanghai 200032, China; E-Mails: kakarwen@163.com (W.-J.W.); luzhongwu0301242@163.com (Z.-W.L.); liduanshu@shca.org.cn (D.-S.L.); wangyu@shca.org.cn (Y.W.); zhuyongxue@shca.org.cn (Y.-X.Z.); wangzhuoyin@shca.org.cn (Z.-Y.W.); wuyi@shca.org.cn (Y.W.)

2 Department of Oncology, Shanghai Medical College, Fudan University, Shanghai 200032, China

$\dagger$ These authors contributed equally to this work.

* Authors to whom correspondence should be addressed;

E-Mails: headneck@126.com (Y.-L.W.); jq_hai@126.com (Q.-H.J.);

Tel.: +86-21-6417-5590 (ext. 2607) (Y.-L.W. \& Q.-H.J.); Fax: +86-21-6417-4774 (Y.-L.W. \& Q.-H.J.).

External Editor: William Chi-shing Cho

Received: 14 August 2014; in revised form: 17 October 2014 / Accepted: 30 October 2014 /

Published: 14 November 2014

\begin{abstract}
Background: The genetic predisposition to papillary thyroid cancer (PTC) is far from clearly elucidated. Rs2292832 is a genetic polymorphism that located in the precursor of mir-149 and has been studied in diverse cancers. Thus far, the role of rs 2292832 in PTC tumorigenesis and progression was unclear; (2) Method: Rs2292832 was genotyped in 838 PTCs, 495 patients with thyroid benign tumors (BNs) and 1006 controls in a Chinese Han population. Clinicopathological data was collected and compared. The expression level of mature mir-149 was examined in 55 normal thyroid tissue samples; (3) Results: The CC genotype of rs2292832 was significantly associated with an increased risk of PTC compared with TT homozygote $(\mathrm{OR}=1.60,95 \% \mathrm{CI}: 1.72-2.20, p=0.003)$ and TT/TC combined genotype (OR $=1.54,95 \%$ CI: $1.14-2.09, p=0.005)$. Rs 2292832 is an independent risk factor correlated with tumor invasion $(p=0.006)$ and higher T stage in PTC patients ( $p=0.007)$, but uncorrelated with short-term disease persistence of PTC. PTC subjects carrying CC genotype have lower mir-149-5p expression than those with TC genotype
\end{abstract}


$(p=0.002)$. Twelve predicted target genes have been identified by collaboratively using computational tools; (4) Conclusion: Rs2292832 was possibly involved in the susceptibility and local progression of PTC in Chinese patients, by altering the expression level of mir-149-5p and its target genes.

Keywords: rs2292832; has-mir-149; PTC susceptibility; clinicopathological characteristics

\section{Introduction}

Papillary thyroid cancer (PTC) is the most common type of thyroid cancer, which is the fifth leading malignancy in females [1]. Although it is widely considered that both genetic and environmental factors contribute to PTC carcinogenesis, the etiology is not well characterized [2]. With the accumulation of evidence from case-control and genome-wide association studies (GWASs) based on different study designs and populations [3-7], it has been postulated that individuals differ in their susceptibility to PTC; however, the genetic predisposition to PTC is far from clearly elucidated.

MicroRNAs are small noncoding RNA molecules, functioning as post-transcriptional suppressors of mRNA translation and being involved in a wide range of cellular processes [8]. The role of microRNA in the tumorigenesis and progression of different types of malignant tumors has been revealed by many previous studies [9-11]. Single nucleotide polymorphism (SNP) located within precursor miRNA (Pre-miRNA) sequence may have an effect on miRNA properties and functions by either affecting the maturation process or altering the target binding. Studies have indicated associations between SNP of miRNA and susceptibility to a variety of cancers [12]. Currently, to our best knowledge, rs2910164 in mir-146a was the only miRNA SNP that have been studied in thyroid cancer. An association between rs2910164 and risk of PTC was found in US and European populations by Jazdzewski K et al., but failed to be replicated in Chinese population and another UK population in recent studies [13-15].

The rs2292832 C/T polymorphism in pre-mir-149 is another pre-miRNA SNP that was studied in diverse cancers. Research results about this SNP are inconsistent. Kim et al. demonstrated that the mir-149 CT and CT/CC genotypes were associated with lower risk of liver cancer, while C allele of hsa-mir-149 may have a potentially protective role in male gastric cancer [16,17]. In spite of these findings, studies failed to detect significant associations between rs2292832 and other malignant tumors, such as lung cancer, colorectal cancer, and head neck squamous cell cancer (HNSCC) [18]. Thus far, the role of mir-149 and rs2292832 in PTC carcinogenesis and progression has been unclear. This study aims to examine the relationship of the pre-mir-149 rs2292832 polymorphism with PTC risk and further evaluate the clinicopathological characteristic of PTC patients with different genotypes in a Chinese population.

\section{Results}

\subsection{Summary of Clinicopathologic Characteristics}

The study population consisted of 838 PTC cases, 495 benign tumor $(\mathrm{BN})$ subjects and 1006 cancer-free controls. As shown in Table 1, no significant differences in age and gender were found between PTC and control groups, while $\mathrm{BN}$ group were significantly older $(48.64 \pm 11.75$ vs. $47.20 \pm 12.29, p=0.03)$ 
and had more females $(74.5 \%$ vs. $69.2 \%, p=0.03)$ compared with control group. Among 838 PTC patients, $387(46.2 \%)$ were diagnosed with microcarcinoma $(\leq 10 \mathrm{~mm})$. Extrathyroidal invasion was present in 138 patients $(16.5 \%)$, including 82 with microscopic infiltration, 49 with moderate advanced gross extension (T3), and 7 with very advanced gross extension (T4). Multifocality and bilateral disease were present in $234(27.9 \%)$ and 151 (18.0\%) cases, respectively. Central and lateral neck lymph node metastases were found in 415 (49.5\%) and 184 (22.0\%) cases. According to American Joint Committee on Cancer tumor node metastasis （AJCC TNM） staging for thyroid cancer (7thed, 2012), 592 (70.7\%), 146 (17.4\%) and 100 (11.9\%) patients were diagnosed at stage I-II, III and IV, respectively. Among 495 cases, there were 299 nodular goiter (60.4\%), 104 follicular adenoma (21.0\%) and 92 cases with both (18.6\%).

Table 1. Characteristics of the study population.

\begin{tabular}{|c|c|c|c|c|c|}
\hline Characteristics & PTC $(n=838)$ & BN $(n=495)$ & Control $(n=1006)$ & $P_{\text {PTC vs. Con }}$ & $P_{\mathrm{BN} v \mathrm{~s} . \mathrm{Con}}$ \\
\hline \multicolumn{6}{|l|}{ Age } \\
\hline$<45$ & $365(43.6)$ & $180(36.4)$ & $435(43.2)$ & 0.89 & 0.01 \\
\hline$\geq 45$ & $473(56.4)$ & $315(63.6)$ & $571(56.8)$ & & \\
\hline Age $($ mean $\pm \mathrm{SD})$ & $46.30 \pm 11.02$ & $48.64 \pm 11.75$ & $47.20 \pm 12.29$ & 0.11 & 0.03 \\
\hline \multicolumn{6}{|l|}{ Gender } \\
\hline Male & $227(27.1)$ & $126(25.5)$ & $310(30.8)$ & 0.08 & 0.03 \\
\hline Female & $611(72.9)$ & $369(74.5)$ & $696(69.2)$ & & \\
\hline \multicolumn{6}{|l|}{ Tumor size } \\
\hline Mean $(\mathrm{cm})$ & $1.26 \pm 0.97$ & - & - & & \\
\hline$\leq 10 \mathrm{~mm}$ & $387(46.2)$ & - & - & & \\
\hline$>10 \mathrm{~mm}$ & $451(53.8)$ & - & - & & \\
\hline \multicolumn{6}{|l|}{ Invasion } \\
\hline Negative & $700(83.5)$ & - & - & & \\
\hline Minimal extension & $82(9.8)$ & - & - & & \\
\hline Advanced disease (T4a) & $49(5.8)$ & - & - & & \\
\hline Advanced disease (T4b) & $7(0.8)$ & - & - & & \\
\hline Multifocal (\%) & $234(27.9)$ & - & - & & \\
\hline Bilateral (\%) & $151(18.0)$ & - & - & & \\
\hline \multicolumn{6}{|l|}{ T stage } \\
\hline $\mathrm{T} 1-\mathrm{T} 2$ & $690(82.3)$ & - & - & & \\
\hline $\mathrm{T} 3-\mathrm{T} 4$ & $148(17.7)$ & - & - & & \\
\hline $\begin{array}{l}\text { Central neck lymph node } \\
\text { metastasis }(\%)\end{array}$ & $415(49.5)$ & - & - & & \\
\hline $\begin{array}{l}\text { Lateral neck lymph node } \\
\text { metastasis }(\%)\end{array}$ & $184(22.0)$ & - & - & & \\
\hline
\end{tabular}

\subsection{Association between Rs2292832 Polymorphism and Risk of Thyroid Tumor}

For rs2292832 polymorphism, Hardy-Weinberg equilibrium was fulfilled in the control group $(p=0.57)$. No inconsistent or abnormal result was found in positive and negative controls, suggesting a good concordance. The genotypic and allelic distributions of rs2292832 in three groups were presented in Table 2. The CC genotype was significantly associated with a 1.60 -fold increased risk 
of PTC compared with TT homozygote $(\mathrm{OR}=1.60,95 \% \mathrm{CI}: 1.72-2.20, p=0.003)$ and TT/TC combined genotype $(\mathrm{OR}=1.54,95 \% \mathrm{CI}: 1.14-2.09, p=0.005)$, after controlling for age and gender. No statistically significant association was observed between $\mathrm{BN}$ and control groups. The frequency of the $\mathrm{C}$ allele was similar with that reported by Hapmap in the $\mathrm{CHB}$ population (MAF $=0.34$ ).

Table 2. Distributions of rs2292832 genotypes and alleles in three groups and their associations with risk of thyroid tumor.

\begin{tabular}{|c|c|c|c|c|c|c|c|}
\hline Rs2292832 & $\begin{array}{l}\text { PTC } \\
(n=838)\end{array}$ & $\begin{array}{l}\text { BN } \\
(n=495)\end{array}$ & $\begin{array}{l}\text { Control } \\
(n=1006)\end{array}$ & $\begin{array}{l}\text { OR }(95 \% \text { CIs })^{a} \\
\text { PTC vs. Con }\end{array}$ & $p$ Value & $\begin{array}{l}\text { OR }(95 \% \text { CIs }) \\
\text { BN vs. Con }\end{array}$ & $p$ Value \\
\hline \multicolumn{8}{|l|}{ Genotype } \\
\hline TT & $379(45.3)$ & $216(43.6)$ & $496(49.3)$ & 1 (reference) & 0.01 & 1 (reference) & 0.12 \\
\hline $\mathrm{TC}$ & $354(42.2)$ & 227 (45.9) & $424(42.1)$ & $1.09(0.90-1.32)$ & 0.41 & $1.22(0.97-1.53)$ & 0.09 \\
\hline $\mathrm{CC}$ & $105(12.5)$ & $52(10.5)$ & $86(8.6)$ & $1.60(1.17-2.20)$ & 0.003 & $1.36(0.93-2.00)$ & 0.11 \\
\hline $\mathrm{TC} / \mathrm{CC}^{\mathrm{D}}$ & $459(54.7)$ & $279(56.4)$ & $510(50.7)$ & $1.17(0.98-1.41)$ & 0.09 & $1.24(1.01-1.55)$ & 0.05 \\
\hline $\mathrm{TT} / \mathrm{TC}^{\mathrm{R}}$ & $733(87.5)$ & $443(89.5)$ & $920(91.4)$ & $1.54(1.14-2.09)$ & 0.005 & $1.23(0.86-1.78)$ & 0.26 \\
\hline \multicolumn{8}{|l|}{ Allele } \\
\hline $\mathrm{T}$ & 0.66 & 0.66 & 0.70 & 1 (reference) & & 1 (reference) & \\
\hline $\mathrm{C}$ & 0.34 & 0.34 & 0.30 & $1.10(1.02-1.18)$ & 0.009 & $1.09(1.00-1.18)$ & 0.05 \\
\hline
\end{tabular}

${ }^{\text {a }}$ Adjusted for age and gender by a logistic regression model.; ${ }^{\mathrm{D}}$ Dominant model: Minor allele containing genotype vs. Major homozygote genotype; ${ }^{\mathrm{R}}$ Recessivemodel: Minor homozygote genotype vs. Major allele containing genotype.

\subsection{Characteristics of Papillary Thyroid Cancer (PTC) Patients with Different Rs2292832 Single} Nucleotide Polymorphism (SNP) Genotypes

As shown in Table 3, univariate analyses showed significant discrepancies of microcarcinoma, exthyroidal invasion, and T stage ( $p=0.032,0.009$ and 0.023 , respectively) among PTC patients with different rs2292832 genotypes, while other features appear to be similar across three groups. A multivariate logistic regression analysis in Table 4 suggests that rs2292832 is probably an independent risk factor correlated with tumor invasion $(p=0.006)$ and higher T stage in PTC patients $(p=0.007)$, adjusting for possible confounders (gender, age, size and lymph node metastasis). The TC heterozygote of rs2292832 SNP was associated with decreased risk of advanced local disease compared with other two genotypes (For invasion: OR $=0.61,95 \% \mathrm{CI}$ : $0.39-0.95, p=0.028$; For T stage: $\mathrm{OR}=0.60,95 \% \mathrm{CI}: 0.37-0.97, p=0.037)$.

Table 3. The clinical characteristics of papillary thyroid cancer (PTC) with different rs2292832 genotypes.

\begin{tabular}{lllll}
\hline Characteristics & $\begin{array}{l}\text { TT Genotype } \\
(\boldsymbol{n}=\mathbf{3 7 9})\end{array}$ & $\begin{array}{l}\text { TC Genotype } \\
(\boldsymbol{n}=\mathbf{3 5 4})\end{array}$ & $\begin{array}{l}\text { CC Genotype } \\
(\boldsymbol{n}=\mathbf{1 0 5})\end{array}$ & $\boldsymbol{p}$ Value a $^{\mathrm{a}}$ \\
\hline Gender & & & & \\
\hline Male & $102(26.9)$ & $97(27.4)$ & $28(26.7)$ & 0.984 \\
Female & $277(73.1)$ & $257(72.6)$ & $77(73.3)$ & \\
\hline Age & & & & 0.569 \\
\hline$<45$ & $170(44.9)$ & $154(43.5)$ & $41(39.0)$ & \\
$\geq 45$ & $209(55.1)$ & $200(56.5)$ & $64(61.0)$ & \\
\hline
\end{tabular}


Table 3. Cont.

\begin{tabular}{|c|c|c|c|c|}
\hline Characteristics & $\begin{array}{l}\text { TT Genotype } \\
(n=379)\end{array}$ & $\begin{array}{l}\text { TC Genotype } \\
(n=354)\end{array}$ & $\begin{array}{l}\text { CC Genotype } \\
(n=105)\end{array}$ & $p$ value $^{a}$ \\
\hline \multicolumn{5}{|l|}{ Size } \\
\hline$\leq 1 \mathrm{~cm}$ & $162(42.7)$ & $182(51.4)$ & $43(41.0)$ & \multirow[t]{2}{*}{0.032} \\
\hline$>1 \mathrm{~cm}$ & $217(57.3)$ & $172(48.6)$ & $62(59.0)$ & \\
\hline Size (cm) & $1.31 \pm 0.99$ & $1.21 \pm 1.00$ & $1.15 \pm 0.76$ & 0.219 \\
\hline \multicolumn{5}{|l|}{ Invasion } \\
\hline Yes & $71(18.7)$ & $43(12.1)$ & $24(22.9)$ & \multirow[t]{2}{*}{0.009} \\
\hline No & $308(81.3)$ & $311(87.9)$ & $81(77.1)$ & \\
\hline \multicolumn{5}{|l|}{ Multifocal } \\
\hline Yes & $109(28.8)$ & $98(27.7)$ & $27(25.7)$ & \multirow[t]{2}{*}{0.820} \\
\hline No & $270(71.2)$ & $256(72.3)$ & $78(74.3)$ & \\
\hline \multicolumn{5}{|l|}{ Bilateral } \\
\hline Yes & $65(17.2)$ & $68(19.2)$ & $18(17.1)$ & \multirow[t]{2}{*}{0.745} \\
\hline No & $314(82.8)$ & $286(80.8)$ & $87(82.9)$ & \\
\hline \multicolumn{5}{|l|}{ With (PTC/BN) } \\
\hline Yes & $45(11.9)$ & $39(11.0)$ & $16(15.1)$ & \multirow[t]{2}{*}{0.503} \\
\hline No & $334(88.1)$ & $315(89.0)$ & $89(84.9)$ & \\
\hline pN+ (Level VI) & $49.6 \%$ & $50.3 \%$ & $46.7 \%$ & 0.808 \\
\hline pN+ (lateral neck) & $23.7 \%$ & $20.6 \%$ & $20.0 \%$ & 0.519 \\
\hline \multicolumn{5}{|l|}{ T stage } \\
\hline I-II & $303(79.9)$ & $306(86.4)$ & $81(77.1)$ & \multirow[t]{2}{*}{0.023} \\
\hline III-IV & $76(20.1)$ & $48(13.6)$ & $24(22.9)$ & \\
\hline
\end{tabular}

Table 4. Multivariate regression analysis for rs2292832 single nucleotide polymorphism (SNP) and local progression features of PTC.

\begin{tabular}{|c|c|c|c|c|c|c|c|c|}
\hline \multirow[b]{2}{*}{ Rs2292832 } & \multicolumn{2}{|c|}{ Invasion } & \multirow[b]{2}{*}{$\begin{array}{l}\text { Odds Ratio }^{\text {a }} \\
(95 \% \text { CI) }\end{array}$} & \multirow[b]{2}{*}{$p$ Value } & \multicolumn{2}{|c|}{ T Stage } & \multirow[b]{2}{*}{$\begin{array}{l}\text { Odds Ratio } \\
(95 \% \text { CI) }\end{array}$} & \multirow[b]{2}{*}{$p$ Value } \\
\hline & $\begin{array}{l}\text { Negative } \\
(n=700)\end{array}$ & $\begin{array}{l}\text { Positive } \\
(n=138)\end{array}$ & & & $\begin{array}{l}\text { I-II } \\
(n=690)\end{array}$ & $\begin{array}{l}\text { III-IV } \\
(n=148)\end{array}$ & & \\
\hline TT & $308(44.0)$ & $71(51.4)$ & 1 (reference) & 0.006 & $303(43.9)$ & $76(51.4)$ & 1 (reference) & 0.007 \\
\hline $\mathrm{TC}$ & $311(44.4)$ & $43(31.2)$ & $\begin{array}{l}0.61 \\
(0.39-0.95)\end{array}$ & & $306(44.3)$ & $48(32.4)$ & $\begin{array}{l}0.60 \\
(0.37-0.97)\end{array}$ & \\
\hline $\mathrm{CC}$ & $81(11.6)$ & $24(17.4)$ & $\begin{array}{l}1.55 \\
(0.89-2.72)\end{array}$ & & $81(11.8)$ & $24(16.2)$ & $\begin{array}{l}1.56 \\
(0.87-2.80)\end{array}$ & \\
\hline $\mathrm{TC} / \mathrm{CC}^{\mathrm{D}}$ & $392(56.0)$ & $67(48.6)$ & $\begin{array}{l}0.79 \\
(0.53-1.18)\end{array}$ & 0.25 & $387(56.1)$ & $72(48.6)$ & $\begin{array}{l}0.81 \\
(0.53-1.21)\end{array}$ & 0.30 \\
\hline $\mathrm{TT} / \mathrm{TC}^{\mathrm{R}}$ & $619(88.4)$ & $114(82.6)$ & $\begin{array}{l}1.93 \\
(1.13-3.28)\end{array}$ & 0.015 & $609(88.2)$ & $124(83.8)$ & $\begin{array}{l}1.92 \\
(1.10-3.35)\end{array}$ & 0.021 \\
\hline Allele C & $33.8 \%$ & $33.0 \%$ & $\begin{array}{l}1.03 \\
(0.89-1.19)\end{array}$ & 0.72 & $33.9 \%$ & $32.4 \%$ & $\begin{array}{l}1.03 \\
(0.88-1.20)\end{array}$ & 0.70 \\
\hline
\end{tabular}

${ }^{\text {a }}$ All of $\mathrm{OR}$ and $p$ values were adjusted for age, gender, tumor size and neck lymph node by a logistic regression model; ${ }^{D}$ Dominant model: Minor allele containing genotype vs. Major homozygote genotype;

${ }^{\mathrm{R}}$ Recessivemodel: Minor homozygote genotype $v s$. Major allele containing genotype. 


\subsection{No Significant Association between Rs2292832 and Short-Term Disease Persistence in PTC Patients}

Patients were followed up until August 2013 with a median follow-up of 35 months, ranging from 17 to 43 months. Thirty-three patients were lost to follow-up and thus were excluded from the analysis. Thirty-four (4.2\%) patients showed persistent disease, including 21 with a recurrence in the lateral neck lymph node compartment, 4 in the ipsilateral VI compartment, and 9 in the thyroid bed. Neither was a significant correlation observed between rs2292832 and short-term disease persistence of PTC (data not shown).

\subsection{Association between Rs2292832 Polymorphism and Expression Level of Mature miR-149} in Thyroid Tissue

To test whether rs 2292832 has any effect on the expression level of mir-149, we selected a subsample of 55 PTC patients, from whom normal thyroid tissue samples were obtained via surgical operations. Of those patients, 20 have TT genotype, 22 have TC genotype, and 13 have CC genotype. Levels of mir-149-5p and mir-149-3p were examined in all 55 normal thyroid tissue samples. As illustrated in Figure 1, PTC subjects carrying CC genotype have lower mir-149-5p expression than those with TC and TT genotype; however, only the difference between CC and TC groups is statistically significant $(p=0.002)$ and an approximate two-fold change was reached. The expression level of mir-149-3p in thyroid tissues was too low to be detected (data not shown).

Figure 1. Association between rs2292832 genotype and expression level of mir-149-5p.



Figure 1 shows scatter spot charts to show the levels of mir-149 expression in normal thyroid tissues obtained from PTC patients with various genotypes. The comparison was performed by using the Mann-Whitney $U$-test. $P_{\mathrm{CC}}$ v. $\mathrm{CT}=0.002 ; P_{\mathrm{CC}}$ s. TT $=0.161$.

\subsection{In Silico Screening for Target Genes of Hsa-mir-149-5p}

To identify mir-149-5p target genes, we performed an in silico screen using five online prediction tools (Targetscan, MiRanda, PicTar, TargetMiner and StarBase). An intersection of predicted target genes between all of five databases was listed in Table 5. Among the 12 predicted genes, ten of them have been studied previously in various types of tumors and were considered as possible roles in diverse 
cancer-related pathways. In terms of thyroid cancer, there were two predicted target genes: PDGFRA, functioning as a tumor progression promoter, and $C D 47$, which may influence the radiation reaction of thyroid cell. Direct relationships between these 12 genes and mir-149-5p were not experimentally proved by far.

Table 5. Predicted most probable target genes of has-mir-149-5p.

\begin{tabular}{|c|c|c|c|c|}
\hline Gene & ID & Full Name & Function & Related Tumor Type \\
\hline$M P Z$ & NM_000530 & Myelin protein zero & Different expression & Bladder cancer \\
\hline PTGIS & NM_000961 & $\begin{array}{l}\text { Prostaglandin } \mathrm{I} 2 \\
\text { (prostacyclin) synthase }\end{array}$ & $\begin{array}{l}\text { Vascular related, } \\
\text { Tumor progression }\end{array}$ & $\begin{array}{l}\text { Colorectal cancer, } \\
\text { Breast cancer }\end{array}$ \\
\hline CREBL2 & NM_001310 & $\begin{array}{l}\text { cAMP responsive element } \\
\text { binding protein-like } 2\end{array}$ & Suppressor gene & $\begin{array}{l}\text { Hematologic } \\
\text { malignancy }\end{array}$ \\
\hline$C D 47$ & NM_001777 & CD47 molecule & Immune and inflammation & $\begin{array}{l}\text { Colorectal cancer } \\
\text { Thyroid cancer }\end{array}$ \\
\hline EIF5 & NM_001969 & $\begin{array}{l}\text { Eukaryotic translation } \\
\text { initiation factor } 5\end{array}$ & Cell proliferation & Leukemia \\
\hline PDGFRA & NM_006206 & $\begin{array}{l}\text { Platelet-derived growth factor } \\
\text { receptor, alpha polypeptide }\end{array}$ & $\begin{array}{l}\text { Oncogene, Tumor } \\
\text { progression }\end{array}$ & $\begin{array}{l}\text { GIST, Gliomas, } \\
\text { Thyroid cancer }\end{array}$ \\
\hline KLHL3 & NM_017415 & $\begin{array}{l}\text { Kelch-like family member } 3 \\
\text { (Drosophila) }\end{array}$ & - & - \\
\hline APPL2 & NM_018171 & $\begin{array}{l}\text { Adaptor protein, } \\
\text { phosphotyrosine interaction, } \\
\text { PH domain and leucine } \\
\text { zipper containing } 2\end{array}$ & Apoptosis & Gliomas \\
\hline$U B F D 1$ & NM_019116 & $\begin{array}{l}\text { Ubiquitin family } \\
\text { domain containing } 1\end{array}$ & - & - \\
\hline CRTC2 & NM_181715 & $\begin{array}{l}\text { CREB regulated } \\
\text { transcription coactivator } 2\end{array}$ & Metabolism & Breast cancer \\
\hline PLAG1 & NM_001114635 & Pleiomorphic adenoma gene 1 & Oncogene & Salivary tumor \\
\hline TOP1 & NM_003286 & Topoisomerase (DNA) I & Cell proliferation & Colorectal cancer \\
\hline
\end{tabular}

\section{Discussion}

To date, associations of four selected SNPs located in an miRNA gene (rs11614913 in hsa-mir-196a2, rs3746444 in hsa-mir-499, rs2910164 in hsa-mir-146a, and rs2292832 in hsa-mir-149) with associated cancer risks have been evaluated by numerous studies [17,19-21]. Among these four SNPs, rs2292832 is the only one located outside the mature region of pre-mir-149 and its reported associations with malignant tumors were inconsistent. In the current study, we first demonstrated a significant difference in terms of genotype distribution of rs2292832 between PTC patients and cancer-free controls. As presented in Table 2, The $\mathrm{CC}$ genotype and $\mathrm{C}$ allele was significantly associated with a 1.60 -fold (95\% CI: $1.72-2.20, p=0.003)$ and a 1.10 -fold (95\% CI: $1.02-1.18, p=0.009$ ) increased risk of PTC, respectively. This is the first study reporting the association between rs2292832 and the risk of PTC; currently, no other comparable studies were conducted in other populations. The distributional difference of rs2292832 genotype frequencies among 
people of various races and ethnicities can be seen in the Hapmap database and previously published researches. The frequencies of $\mathrm{C}$ allele of rs2292832 were much lower in Asian populations than those in other populations (present study: 0.30; CHB: 0.34; ASW: 0.71; CEU: 0.73). Thus, to apply the conclusion of the present study to other racial or ethnic people should be done with caution.

In terms of other types of malignant tumor, rs2292832 has been shown to be associated with risks of hepatocellular carcinoma and gastric cancer, however the findings favor CC/TC genotype and C allele as a protective role which differs from ours [16,17]. This divergence is probably not attributable to genetic diversity of different ethnic populations, since those studies with opposite results were also conducted in Asian populations, having similar genotype distributions as the present one. Thus, one possible explanation for this discrepancy is the effect of rs2292832 on cancer susceptibility may vary according to different tumor types.

To examine whether rs2292832 had any effect on biological behaviors of PTC, we compared clinicopathologic characteristics among PTC patients with three different genotypes. CC genotype of rs2292832 SNP was associated with increased risk of PTC as well as more advanced local tumor progression, while TC heterozygote plays a protective role. A possible explanation was that although tumor sizes were similar between three genotype groups, the proportion of microcarcinoma, which have more indolent behavior than PTC large than $10 \mathrm{~mm}$, was more prevalent in PTC patients with TC genotype. In fact, when we performed the multivariate regression analysis adjusting for tumor size as a dichotomous variable ( $\leq 10 \mathrm{~mm} v s .>10 \mathrm{~mm}$ ) instead of as a continuous one, the associations of TC genotype with decreased risk of invasion and higher T stage appeared not significant $(p>0.05)$. In sum, our results suggest that rs2292832 and has-mir-149 may be involved in the processes of PTC tumorigensis and local progression.

Current laboratory research supposes that SNPs in sequence of pre-miRNA influence the function of miRNA through two main mechanisms: (1) Change the sequence specificity of miRNA core regions and alter the combining affinity between miRNA and mRNA; (2) Influence the maturation process of miRNA and alter its expression level. Since rs2292832 is located outside the seed region in mature mir-149, the latter mechanism may be more appropriate to explain the effect of rs2292832 on mir-149. Using RT-PCR technology, we evaluated the expression level of mature mir-149 in normal thyroid tissues collected from PTC patients with three rs2292832 genotypes. Results showed that mir-149-5p was the dominant form of mature has-mir-149 in thyroid tissue rather than mir-149-3p. In addition, PTC subjects carrying the CC genotype have lower mir-149-5p expression level than those with TC and TT genotypes. This result was inconsistent with that of another previous study, which found HNSCC patients carrying TT and TC genotypes tended to express lower but statistically non-significant level of miR-149 [22]. Despite this inconsistence, what the expression study measured is in concordance with our findings from the aforementioned association analysis and confirms our hypothesis to some extent; rs2292832 CC homozygote affects PTC susceptibility and local tumor progression through altering the expression level of mature mir-149-5p and probably further regulating the translation of its downstream target genes. Functional in vitro study was required to test this hypothesis, however.

Because CC genotype of rs2292832 was simultaneously associated with higher risk of PTC tumorigenesis and invasion, as well as with lower expression level of mir-149, we can propose that mir-149 may function as a tumor suppressor. This speculation was partly in line with results from 
a recent study, in which Bischoff et al. found miR-149 down-regulation facilitates the cell spreading, migration and invasion of basal-like breast cancer cells [23]. Currently, the experimentally validated target gene of mir-149-5p was still not well examined. SRPX2 and FOXM1 were proven to be directly regulated by mir-149 in colorectal cancer and lung cancer, respectively [24,25]. In this study, we predicted the mir-149 target gene computationally by using several online databases and tools; an intersection including twelve genes from five mainstream databases was established. Among these 12 genes, ten were cancer-related, as revealed by previous reports, while eight were found to function as oncogene and tumor promoter. Therefore, as a negative regulator of these genes, mir-149 is more likely to be a tumor suppressor, which again is concordant with other results in this study.

Among twelve predicted genes, PDGFRA and CD47 had been studied in thyroid cancer [26,27]. PDGFRA encodes a cell surface tyrosine kinase receptor for members of the platelet-derived growth factor family. Studies suggested that this gene plays a role in organ development, wound healing, and tumor progression. Mutations, copy number variations, and polymorphisms in this gene have been found to be associated with somatic and familial gastrointestinal stromal tumors (GIST), as well as other cancers [28-31]. Recently, cumulating experimental evidence showed that high expression level of PDGFRA was associated with increased invasive and migration potential of PTC. Downstream up-regulation of both MAPK/ERK and PI3K/Akt pathways was observed after activation of PDGFRA in PTC cell line [26]. Although Guan et al. reported that down-regulation of mir-218-2 could promote the invasion and progression of thyroid cancer partially by targeting PDGFRA, the mechanism of PDGFRA up-regulation in PTC tissue was not completely clear by far [32]. In addition, another study found PDGFRA promoter SNPs (rs6554162 and rs1800812) may be associated with the risk of PTC [27]. Thus, PDGFRA is probably an appropriate candidate target gene which can be helpful to elucidate roles of rs2292832 and mir-149 in PTC initiation and progression in future studies. The correlation between CD47 and PTC was reported previously in only one paper, in which authors found that after treated with Iodine-131, the expression of $C D 47$ were different in normal thyroid tissues, suggesting a causal relationship between $C D 47$ expression and radiation reaction in thyroid cell [33]. However, no further functional evidence have emerged to support this hypothesis.

In summary, this study suggested that rs2292832 is possibly associated with the susceptibility and exthyroidal invasion of PTC in Chinese patients. This effect of rs2292832 may be affected by the altered expression of mature mir-149-5p which is down-regulated in patients with CC genotype of rs2292832, as compared with other two genotypes. Twelve predicted target genes have been identified by collaboratively using computational tools. Because of a racially non-diverse sample, the application of this conclusion to other populations is restricted and further validation studies based on a multi-race population is needed. Meanwhile, to further elucidate roles of rs2292832 and mir-149 in PTC initiation and progression, experimentally functional studies are required in future.

\section{Materials and Methods}

\subsection{Study Subjects}

The study population has been described in detail in previous publications [34]. Of a total 2339 study subjects, 838 are PTC patients, 495 are BN patients and, 1006 are cancer-free controls (8 PTC cases 
and $11 \mathrm{BN}$ cases were excluded because of incomplete clinical data or failed genotyping). All subjects are ethnically Chinese Han and from Eastern China. PTC and BN cases were enrolled from those who were treated at the Department of Head and Neck Surgery of Cancer Hospital, Fudan University, in Shanghai from January to December 2010. Patients with a confirmed histological diagnosis of PTC or BN were included, while those with history of thyroid disease treatment and radiation exposure were excluded. Cancer-free controls without history of any kind of thyroid disease were randomly selected from the Taizhou Longitudinal Study during the same period [35]. In addition, tissue samples were obtained from 55 PTC patients who have undergone surgical operation in the Department of Head and Neck Surgery. This study was approved by the Ethical Committee of Cancer Hospital, Fudan University, and informed consent was obtained from all subjects.

\subsection{DNA Extraction and Genotyping}

About 3-5 mL venous blood was collected from each subject. The Qiagen Blood Kit (Qiagen, Chatsworth, CA, USA) was used to extract genomic DNA. Genotyping was performed with the MassARRAY iPLEX platform (Sequenom Inc., San Diego, CA, USA) using an allele-specific matrix-assisted laser desorption/ionization time of flight mass spectrometry assay (MALDI-TOF). Reagents for genotyping were acquired from iPLEX ${ }^{\circledR}$ Gold Reagent Kit (Sequenom Inc., San Diego, CA, USA). Primers for amplification and extension reactions were designed using MassARRAY Assay Design software. Results of the genotyping were read and output by TYPER 4.0 software (Sequenom Inc., San Diego, CA, USA). For better quality control, each plate of samples contained at least four internal positive controls of DNA samples randomly selected in the same plate and two negative controls of pure water. Operators who performed the genotyping assays were blind to the study group of each sample.

\section{3. qRT-PCR Analysis}

Total RNA was extracted from frozen tissue using the miRVana miRNA Isolation Kit and was reverse-transcribed using a specific stem-loop RT primer (Applied Biosystems, Foster City, CA, USA). To quantify the expression level of mir-149, a TaqMan miRNA assay kit was also purchased from Applied Biosystems. Experimental operations were performed according to the manufacturer's instructions. RNU6B was employed as internal controls and water was employed as negative controls. Quantitative PCR was carried out using an Applied Biosystems 7900 HT real-time PCR system. Each sample was analyzed in triplicate. Thermal cycling was initiated with an hold step for $10 \mathrm{~min}$ at $95^{\circ} \mathrm{C}$, followed by 40 cycles of $95^{\circ} \mathrm{C}$ for $15 \mathrm{~s}$ and $60{ }^{\circ} \mathrm{C}$ for $60 \mathrm{~s}$. Data were analyzed with ABISDS version 2.3. To examine the quality of the results, operators who performed the qRT-PCR assays were unaware of the study group of each sample. $C_{\mathrm{T}}$ values of mir-149 and RNU6B were obtained for all samples. The normalized expression level of each sample was designated as $-\Delta C$, and was the difference in $C_{\mathrm{T}}$ values between the RNU6B and mir-149. $2^{-\Delta \Delta C T}$ represents the fold change of mir-149 expression in patients with different rs2292832 genotype. 


\subsection{Clinical Management}

The management schema of thyroid nodules in our center was described previously [34]. Briefly, all patients received an ultrasound examination prior to surgery. Fine needle aspiration (FNA) and computed tomography scans were performed individually. A lobectomy with a pathological frozen section examination was routinely performed during the operation. When a benign or undetermined nodule was detected in the contralateral lobe by Ultrasound (US), a subtotal lobectomy with frozen section was also initially performed. If malignant lesions were found in both lobes of the thyroid by frozen section, a total thyroidectomy plus a bilateral level VI lymph node dissection was performed. A lateral neck dissection was performed only in cases with clinically evident lateral neck lymph node metastasis.

Postsurgical radioactive iodine therapy was limited to patients who had distant metastases because its use is strictly controlled in China. All PTC subjects received thyroid stimulating hormone (TSH)-suppressive hormonal therapy after surgery. During the follow-up period, all patients were suggested to receive US examination of the thyroid and neck every 3-6 months in the first year, 6-12 months in the 2-3 years after operation, and then annually if it they were disease-free. Locoregional recurrence was diagnosed by US or Computed Tomography (CT) plus FNA when needed.

\subsection{Statistical Analysis}

Distributional differences of selected variables and Hardy-Weinberg equilibrium between cases and controls were evaluated using the Chi-square test and Student's $t$ test as appropriate. The associations of each SNP with PTC risk and clinicopathologic characteristics were measured as odds ratio (OR) and its 95\% confidence interval (CI), using multivariate logistic regression analyses. In the multivariate analyses, the common homozygote genotype was used as the reference category. A $p$-value $<0.05$ was considered statistically significant. All the statistical analyses were performed with SPSS software, version 12.0 (SPSS, Chicago, IL, USA).

\section{Acknowledgments}

This work was supported by the National Science Foundation of China (81001204 to Yu-Long Wang, 30872958 to Qing-Hai Ji and 30971594 to Jiu-Cun Wang).

\section{Author Contributions}

Wen-Jun Wei, Yu-Long Wang and Qing-Hai Ji conceived and designed the experiments; Zhong-Wu Lu and Yu Wang performed the experiments; Wen-Jun Wei and Zhuo-Ying Wang analyzed the data; Duan-shu Li, Yong-xue Zhu and Yi Wu contributed reagents/materials/analysis tools; Wen-Jun Wei and Zhong-Wu Lu wrote the paper.

\section{Conflicts of Interest}

The authors declare no conflict of interest. 


\section{References}

1. Siegel, R.; Naishadham, D.; Jemal, A. Cancer statistics, 2013. CA Cancer J. Clin. 2013, 63, 11-30.

2. Kondo, T.; Ezzat, S.; Asa, S.L. Pathogenetic mechanisms in thyroid follicular-cell neoplasia. Nat. Rev. Cancer 2006, 6, 292-306.

3. Adjadj, E.; Schlumberger, M.; de Vathaire, F. Germ-line DNA polymorphisms and susceptibility to differentiated thyroid cancer. Lancet Oncol. 2009, 10, 181-190.

4. Gudmundsson, J.; Sulem, P.; Gudbjartsson, D.F.; Jonasson, J.G.; Sigurdsson, A.; Bergthorsson, J.T.; He, H.; Blondal, T.; Geller, F.; Jakobsdottir, M.; et al. Common variants on 9q22.33 and 14q13.3 predispose to thyroid cancer in European populations. Nat. Genet. 2009, 41, 460-464.

5. Gudmundsson, J.; Sulem, P.; Gudbjartsson, D.F.; Jonasson, J.G.; Masson, G.; He, H.; Jonasdottir, A.; Sigurdsson, A.; Stacey, S.N.; Johannsdottir, H.; et al. Discovery of common variants associated with low TSH levels and thyroid cancer risk. Nat. Genet. 2012, 44, 319-322.

6. Matsuse, M.; Takahashi, M.; Mitsutake, N.; Nishihara, E.; Hirokawa, M.; Kawaguchi, T.; Rogounovitch, T.; Saenko, V.; Bychkov, A.; Suzuki, K.; et al. The FOXE1 and NKX2-1 loci are associated with susceptibility to papillary thyroid carcinoma in the Japanese population. J. Med. Genet. 2011, 48, 645-648.

7. Kohler, A.; Chen, B.; Gemignani, F.; Elisei, R.; Romei, C.; Figlioli, G.; Cipollini, M.; Cristaudo, A.; Bambi, F.; Hoffmann, P.; et al. Genome-wide association study on differentiated thyroid cancer. J. Clin. Endocrinol. Metab. 2013, 98, E1674-E1681.

8. Bartel, D.P. MicroRNAs: Genomics, biogenesis, mechanism and function. Cell 2004, 116, 281-297.

9. Lu, J.; Getz, G.; Miska, E.A.; Alvarez-Saavedra, E.; Lamb, J.; Peck, D.; Sweet-Cordero, A.; Ebert, B.L.; Mak, R.H.; Ferrando, A.A.; et al. MicroRNA expression profiles classify human cancers. Nature 2005, 435, 834-838.

10. Hu, Z.; Chen, X.; Zhao, Y.; Tian, T.; Jin, G.; Shu, Y.; Chen, Y.; Xu, L.; Zen, K.; Zhang, C.; Shen, H. Serum microRNA signatures identified in a genome-wide serum microRNA expression profiling predict survival of non-small-cell lung cancer. J. Clin. Oncol. 2010, 28, 1721-1726.

11. Ji, J.; Shi, J.; Budhu, A.; Yu, Z.; Forgues, M.; Roessler, S.; Ambs, S.; Chen, Y.; Meltzer, P.S.; Croce, C.M.; et al. MicroRNA expression, survival, and response to interferon in liver cancer. N. Engl. J. Med. 2009, 361, 1437-1447.

12. Ryan, B.M.; Robles, A.I.; Harris, C.C. Genetic variation in microRNA networks: The implications for cancer research. Nat. Rev. Cancer 2010, 10, 389-402.

13. Jazdzewski, K.; Murray, E.L.; Franssila, K.; Jarzab, B.; Schoenberg, D.R.; de la Chapelle, A. Common SNP in pre-mir-146a decreases mature mir expression and predisposes to papillary thyroid carcinoma. Proc. Natl. Acad. Sci. USA 2008, 105, 7269-7274.

14. Wei, W.J.; Wang, Y.L.; Li, D.S.; Wang, Y.; Wang, X.F.; Zhu, Y.X.; Yang, Y.J.; Wang, Z.Y.; Ma, Y.Y.; Wu, Y.; et al. Association between the rs2910164 polymorphism in pre-mir-146a sequence and thyroid carcinogenesis. PLoS One 2013, 8, e56638.

15. Jones, A.M.; Howarth, K.M.; Martin, L.; Gorman, M.; Mihai, R.; Moss, L.; Auton, A.; Lemon, C.; Mehanna, H.; Mohan, H.; et al. Thyroid cancer susceptibility polymorphisms: Confirmation of loci on chromosomes $9 \mathrm{q} 22$ and 14q13, validation of a recessive 8q24 locus and failure to replicate a locus on 5q24. J. Med. Genet. 2012, 49, 158-163. 
16. Kim, W.H.; Min, K.T.; Jeon, Y.J.; Kwon, C.I.; Ko, K.H.; Park, P.W.; Hong, S.P.; Rim, K.S.; Kwon, S.W.; Hwang, S.G.; et al. Association study of microRNA polymorphisms with hepatocellular carcinoma in Korean population. Gene 2012, 504, 92-97.

17. Ahn, D.H.; Rah, H.; Choi, Y.K.; Jeon, Y.J.; Min, K.T.; Kwack, K.; Hong, S.P.; Hwang, S.G.; Kim, N.K. Association of the mir-146aC $>$ G, mir-149T $>C$, mir-196a2T $>C$, and mir-499A $>\mathrm{G}$ polymorphisms with gastric cancer risk and survival in the Korean population. Mol. Carcinog. 2013, 52, E39-E51.

18. Xu, L.; Zhou, X.; Qiu, M.T.; Yin, R.; Wu, Y.Q.; Xu, L. Lack of association between hsa-mir-149 rs2292832 polymorphism and cancer risk: A meta-analysis of 12 studies. PLoS One 2013, 8, e73762.

19. Min, K.T.; Kim, J.W.; Jeon, Y.J.; Jang, M.J.; Chong, S.Y.; Oh, D.; Kim, N.K. Association of the mir-146aC $>\mathrm{G}, 149 \mathrm{C}>\mathrm{T}, 196 \mathrm{a} 2 \mathrm{C}>\mathrm{T}$, and $499 \mathrm{~A}>\mathrm{G}$ polymorphisms with colorectal cancer in the Korean population. Mol. Carcinog. 2012, 51, E65-E73.

20. Hu, Z.; Liang, J.; Wang, Z.; Tian, T.; Zhou, X.; Chen, J.; Miao, R.; Wang, Y.; Wang, X.; Shen, H. Common genetic variants in pre-microRNAs were associated with increased risk of breast cancer in Chinese women. Hum. Mutat. 2009, 30, 79-84.

21. Liu, Z.; Li, G.; Wei, S.; Niu, J.; El-Naggar, A.K.; Sturgis, E.M.; Wei, Q. Genetic variants in selected pre-microRNA genes and the risk of squamous cell carcinoma of the head and neck. Cancer 2010, 116, 4753-4760.

22. Tu, H.F.; Liu, C.J.; Chang, C.L.; Wang, P.W.; Kao, S.Y.; Yang, C.C.; Yu, E.H.; Lin, S.C.; Chang, K.W. The association between genetic polymorphism and the processing efficiency of mir-149 affects the prognosis of patients with head and neck squamous cell carcinoma. PLoS One 2012, 7, e51606.

23. Bischoff, A.; Huck, B.; Keller, B.; Strotbek, M.; Schmid, S.; Boerries, M.; Busch, H.; Muller, D.; Olayioye, M.A. miR-149 functions as a tumor suppressor by controlling breast epithelial cell migration and invasion. Cancer Res. 2014, 74, 5256-5265.

24. Oster, B.; Linnet, L.; Christensen, L.L.; Thorsen, K.; Ongen, H.; Dermitzakis, E.T.; Sandoval, J.; Moran, S.; Esteller, M.; Hansen, T.F.; et al. Non-CpG island promoter hypomethylation and mir-149 regulate the expression of SRPX2 in colorectal cancer. Int. J. Cancer 2013, 132, 2303-2315.

25. Ke, Y.; Zhao, W.; Xiong, J.; Cao, R. miR-149 inhibits non-small-cell lung cancer cells EMT by targeting FOXM1. Biochem. Res. Int. 2013, 2013, 506731.

26. Zhang, J.; Wang, P.; Dykstra, M.; Gelebart, P.; Williams, D.; Ingham, R.; Adewuyi, E.E.; Lai, R.; McMullen, T. Platelet-derived growth factor receptor- $\alpha$ promotes lymphatic metastases in papillary thyroid cancer. J. Pathol. 2012, 228, 241-250.

27. Kim, M.J.; Kim, S.K.; Park, H.J.; Chung, D.H.; Park, H.K.; Lee, J.S.; Kwon, K.H.; Chung, J.H. PDGFRA promoter polymorphisms are associated with the risk of papillary thyroid cancer. Mol. Med. Rep. 2012, 5, 1267-1270.

28. Nannini, M.; Biasco, G.; Astolfi, A.; Pantaleo, M.A. An overview on molecular biology of KIT/PDGFRA wild type (WT) gastrointestinal stromal tumours (GIST). J. Med. Genet. 2013, 50, 653-661. 
29. Velghe, A.I.; van Cauwenberghe, S.; Polyansky, A.A.; Chand, D.; Montano-Almendras, C.P.; Charni, S.; Hallberg, B.; Essaghir, A.; Demoulin, J.B. PDGFRA alterations in cancer: Characterization of a gain-of-function V536E transmembrane mutant as well as loss-of-function and passenger mutations. Oncogene 2014, 33, 2568-2576.

30. Silva, M.; Veiga, I.; Ribeiro, F.R.; Vieira, J.; Pinto, C.; Pinheiro, M.; Mesquita, B.; Santos, C.; Soares, M.; Dinis, J.; et al. Chromosome copy number changes carry prognostic information independent of KIT/PDGFRA point mutations in gastrointestinal stromal tumors. BMC Med. 2010, $8,26$.

31. Verhaak, R.G.; Hoadley, K.A.; Purdom, E.; Wang, V.; Qi, Y.; Wilkerson, M.D.; Miller, C.R.; Ding, L.; Golub, T.; Mesirov, J.P.; et al. Integrated genomic analysis identifies clinically relevant subtypes of glioblastoma characterized by abnormalities in PDGFRA, IDH1, EGFR, and NF1. Cancer Cell 2010, 17, 98-110.

32. Guan, H.; Wei, G.; Wu, J.; Fang, D.; Liao, Z.; Xiao, H.; Li, M.; Li, Y. Down-regulation of mir-218-2 and its host gene SLIT3 cooperate to promote invasion and progression of thyroid cancer. J. Clin. Endocrinol. Metab. 2013, 98, E1334-E1344.

33. Abend, M.; Pfeiffer, R.M.; Ruf, C.; Hatch, M.; Bogdanova, T.I.; Tronko, M.D.; Hartmann, J.; Meineke, V.; Mabuchi, K.; Brenner, A.V. Iodine-131 dose-dependent gene expression: Alterations in both normal and tumour thyroid tissues of post-Chernobyl thyroid cancers. $\mathrm{Br}$. $J$. Cancer 2013, 109, 2286-2294.

34. Wang, Y.L.; Feng, S.H.; Guo, S.C.; Wei, W.J.; Li, D.S.; Wang, Y.; Wang, X.; Wang, Z.Y.; Ma, Y.Y.; Jin, L.; et al. Confirmation of papillary thyroid cancer susceptibility loci identified by genome-wide association studies of chromosomes 14q13, 9q22, 2q35 and 8p12 in a Chinese population. J. Med. Genet. 2013, 50, 689-695.

35. Wang, X.; Lu, M.; Qian, J.; Yang, Y.; Li, S.; Lu, D.; Yu, S.; Meng, W.; Ye, W.; Jin, L. Rationales, design and recruitment of the Taizhou Longitudinal Study. BMC Public Health 2009, 9, 223.

(C) 2014 by the authors; licensee MDPI, Basel, Switzerland. This article is an open access article distributed under the terms and conditions of the Creative Commons Attribution license (http://creativecommons.org/licenses/by/4.0/). 